Prons

nistomention

Journal of Nonlinear Mathematical Physics

\title{
Generalized Wronskian and Grammian Solutions to a Isospectral B- type Kadomtsev-Petviashvili equation
}

Jianjun Cheng, Zhen Wang, Hongqing Zhang

To cite this article: Jianjun Cheng, Zhen Wang, Hongqing Zhang (2014) Generalized Wronskian and Grammian Solutions to a Isospectral B-type Kadomtsev-Petviashvili equation, Journal of Nonlinear Mathematical Physics 21:1, 17-33, DOI:

https://doi.org/10.1080/14029251.2014.894718

To link to this article: https://doi.org/10.1080/14029251.2014.894718

Published online: 04 January 2021 


\title{
Generalized Wronskian and Grammian Solutions to a Isospectral B-type Kadomtsev-Petviashvili equation
}

\author{
Jianjun Cheng \\ School of Mathematical Sciences, Dalian University of Technology \\ Dalian 116024, Liaoning, P. R. China \\ chengjianjun0355@126.com \\ Zhen Wang \\ School of Mathematical Sciences, Dalian University of Technology \\ Dalian 116024, Liaoning, P. R. China \\ wangzhen@dlut.edu.cn \\ Hongqing Zhang \\ School of Mathematical Sciences, Dalian University of Technology \\ Dalian 116024, Liaoning, P. R. China \\ zhanghq@dlut.edu.cn
}

Received 13 June 2013

Accepted 18 November 2013

\begin{abstract}
Generally speaking, the BKP hierarchy which only has Pfaffian solutions. In this paper, based on the Grammian and Wronskian derivative formulae, generalized Wronskian and Grammian determinant solutions are obtained for the isospectral BKP equation (the second member on the BKP hierarchy) in the Hirota bilinear form. Especially, with the help of the properties of the computing of Young diagram, we have first applied Young diagram proved the proposition of this paper. Moreover, by considering the different combinations of the entries in Wronskian, we obtain various types of Wronskian solutions.
\end{abstract}

Keywords: isospectral BKP equation; Wronskian determinant; Grammian determinant; Young diagram.

2000 Mathematics Subject Classification: 76B25, 35G20, 37C07, 37C08, 37C09

\section{Introduction}

With the development of nonlinear science, nonlinear evolution equations (NLEEs) have become attractive in the theoretical and experimental studies because of their potential applications in such fields as fluid dynamics, plasma physics, astrophysics $[2,5,10]$. Nonlinear phenomena play a fundamental role in applied mathematics and physics. The investigation of the exact traveling wave solutions to the NLEEs plays an important role in the study of nonlinear physical phenomena. To understand the mechanisms of those physical phenomena, it is necessary to explore their solutions and properties $[3,4,16,19]$. Consequently, there have been some methods proposed to deal with the NLEEs, such as the inverse scattering transformation (IST) [1], Bäcklund transformation (BT) [20], Darboux transformation (DT) [17], Hirota method [11,12], Wronskian techniques [9, 13, 18, 22, 28], and others. Among those methods, the Wronskian formulations are a common feature for certain NLEEs through the dependent variable transformation [12]. 
The BKP hierarchy (KP hierarchy of B-type) was introduced by Date, Jimbo, Kashiwara, and Miwa [7, 8], in 1981. In 2003, Chen etal [6] constructed the symmetries and algebraic structures for isospectral and nonisospectral BKP system associated with the linear problem of Sato theory. The isospectral B-type Kadomtsev-Petviashvili (isospectral BKP) [14,15] equation reads as:

$$
9 u_{t}-5 u_{x x y}+u_{x x x x}+15 u_{x} u_{x x}+15 u u_{x x x}-15 u u_{y}+45 u^{2} u_{x}-5 \int u_{y y} d x-15 u_{x} \int u_{y} d x=0,
$$

notice that the Eq.(1.1) has the Hirota bilinear equation

$$
\left(D_{x}^{6}-5 D_{x}^{3} D_{y}-5 D_{y}^{2}+9 D_{x} D_{t}\right) \tau \cdot \tau=0,
$$

under the Cole Hopf transformation

$$
u=2(\ln \tau)_{x x},
$$

where the operators D are called Hirota's operators and defined by

$$
D_{x}^{m} D_{y}^{n} f \cdot g=\left.\left(\partial_{x}-\partial_{x^{\prime}}\right)^{m}\left(\partial_{y}-\partial_{y^{\prime}}\right)^{n} f(x, y) \cdot g\left(x^{\prime}, y^{\prime}\right)\right|_{x=x^{\prime}, y=y^{\prime}} .
$$

When $u_{y}=0$ Eq.(1.1) become Sawada-Kotera equation [23]. When $u_{t}=0$ Eq.(1.1) become Ramani equation [21]. When $t \rightarrow \frac{1}{9 b} t+\frac{2 a^{2}}{45 b^{2}} x, y \rightarrow-\frac{a}{5 b} x$ Eq.(1.1) become Sawada-Kotera equation with a nonvanishing boundary condition as [24].

The isospectral BKP equation is the second member on the BKP hierarchy. The Pfaffian solutions to the BKP hierarchy have been given by Hirota $[12,14]$, however, to our knowledge BKP equation (the first member on the BKP hierarchy) which only has Pfaffian solutions and the Wronskian and Grammian solutions to the isospectral BKP equation have not given. In this paper, based on the Wronskian and Grammian derivative formulae, generalized Wronskian and Grammian solutions are obtained for the isospectral BKP equation in the Hirota bilinear form. So the isospectral BKP equation not only has Pfaffian solutions but also has Wronskian and Grammian determinant solutions. With the help of the properties of the computing of Young diagram, we have first applied Young diagram proved the proposition of this paper.

The organization of this paper is as follows: In Section 2, we give three generalized linear differential conditions for Wronskian solution of Eq.(1.2) and under the properties of the Young diagram and the linear differential conditions we have proved the proposition. In Section 3, by considering the different combinations of the entries in Wronskian, we obtain various types of Wronskian solutions, some sample lower-order solutions's figures to describe the propagation of the solitary waves. In Section 4, we give the Grammian determinant solutions of the isospectral BKP equation. Finally, conclusions will be given in Section 5.

\section{The linear differential conditions of Wronskian solutions}

We use the Wronskian technique in the compact notation introduced by Freeman and Nimmo [9]

$$
\tau=\left|\begin{array}{cccr}
\phi_{1}^{(0)} & \phi_{1}^{(1)} & \cdots & \phi_{1}^{(N-1)} \\
\phi_{2}^{(0)} & \phi_{2}^{(1)} & \cdots & \phi_{2}^{(N-1)} \\
\vdots & \vdots & \ddots & \vdots \\
\phi_{N}^{(0)} & \phi_{N}^{(1)} & \cdots & \phi_{N}^{(N-1)}
\end{array}\right|,
$$


where

$$
\phi_{i}^{(j)}=\frac{\partial^{j} \phi_{i}}{\partial x^{j}}, i=1 \ldots N, j=0 \ldots N-1 .
$$

Solutions determined by $\tau=\widehat{N-1 \mid}$ to Eq.(1.1) are called Wronskian determinant solution.

The following sum of products of second-order determinants

$$
\left|\begin{array}{ll}
a_{0} & a_{1} \\
b_{0} & b_{1}
\end{array}\right|\left|\begin{array}{ll}
a_{2} & a_{3} \\
b_{2} & b_{3}
\end{array}\right|-\left|\begin{array}{ll}
a_{0} & a_{2} \\
b_{0} & b_{2}
\end{array}\right|\left|\begin{array}{ll}
a_{1} & a_{3} \\
b_{1} & b_{3}
\end{array}\right|+\left|\begin{array}{ll}
a_{0} & a_{3} \\
b_{0} & b_{3}
\end{array}\right|\left|\begin{array}{ll}
a_{1} & a_{2} \\
b_{1} & b_{2}
\end{array}\right|=0
$$

is satisfied identically. Note that (2.2) can be expressed entirely in terms of the column vectors $c_{i}=\left(a_{i}, b_{i}\right)^{T}$ as

$$
\left|c_{0}, c_{1}\right|\left|c_{2}, c_{3}\right|-\left|c_{0}, c_{2}\right|\left|c_{1}, c_{3}\right|+\left|c_{0}, c_{3}\right|\left|c_{1}, c_{2}\right|=0 \text {. }
$$

This is the simplest case of a Plücker relation. Equation (2.3) can be extended to

$$
\left|C, c_{N-2}, c_{N-1}\right|\left|C, c_{N}, c_{N+1}\right|-\left|C, c_{N-2}, c_{N}\right|\left|C, c_{N-1}, c_{N+1}\right|+\left|C, c_{N-2}, c_{N+1}\right|\left|C, c_{N-1}, c_{N}\right|=0
$$

where $C=c_{0}, c_{1}, \ldots, c_{N-3}$. In fact, only the indices are important in (2.4), and so we may also express it as

$$
\begin{aligned}
& |\widehat{N-1}||\widehat{N-3}, N, N+1|-|\widehat{N-2}, N||\widehat{N-3}, N-1, N+1| \\
& +|\widehat{N-2}, N+1||\widehat{N-3}, N-1, N|=0 .
\end{aligned}
$$

Maya diagrams were first introduced by Mikio Sato [25]. In the language of physics, the diagram for $\tau$ represents the vacuum state in which fermions occupy cells $0,1,2, \cdots, N-2, N-1$. So the Wronskian $\tau=|\widehat{N-1}|$ is expressed with the vacuum state

$$
\tau=\frac{\mathrm{N}-3 \mathrm{~N}-2 \mathrm{~N}-1 \mathrm{~N} \mathrm{~N}+1 \mathrm{~N}+2 \cdots}{\cdots}=\emptyset .
$$

There is a one to one correspondence between the Maya diagram and the Young diagram [12]. Each occupied cell in the Maya diagram corresponds to a vertical line $\uparrow(\bullet)$ in a Young diagram, and the cell correspond to the horizontal line $\rightarrow(0)$ are empty. By using the correspondence between Maya diagrams and Young diagrams, the above Plücker relation (2.5) is expressed using Young diagrams as

$$
\emptyset \times \boxminus-\square \times \boxminus+\square \times \boxminus=0 .
$$

The derivative with respect to $x$ of the wronskian $\tau=\widehat{|N-1|}$ is equal to the sum of determinants $|0,1, \ldots, i+1, \ldots, N-1|$, for $i=0,1, \ldots, N-1$, in which the $i$ th column of $\tau$ is replaced by its derivative. However, the derivative of the first column is equal to the second, the derivative of the second one equals the third, and so on. That is, if we differentiate $\tau$, only the number of derivatives in each column can change, the rows are unaffected.

From $\frac{\partial \phi_{i}}{\partial x_{n}}=\frac{\partial^{n} \phi_{i}}{\partial x^{n}}$, the derivative with respect to $x_{n}$ of the wronskian $\tau=|\widehat{N-1}|$ is equal to the sum of determinants $|0,1, \ldots, i+n, \ldots, N-1|$, for $i=0,1, \ldots, N-1$, in which the $i$ th column of $\tau$ is replaced by its derivative. We observe the rule that the first derivative of the Wronskian with respect to $x_{n}$ is given by a sum of all possible "n-moves" of particles in the vacuum state. It is meant by "n-moves" that a particle moves to the right by $\mathrm{n}$ cells at once. By using the correspondence 
between Maya diagrams and Young diagrams, we have shown that the derivatives of the Wronskian with respect to $x_{n}$ are expressed with the Young diagrams.

$$
\begin{aligned}
& (\emptyset)_{x_{1}}=\square \\
& (\emptyset)_{x_{2}}=-\boxminus+\square \\
& (\emptyset)_{x_{3}}=\text { 目一而 } \\
& (\emptyset)_{x_{4}}=-\boxminus+\boxminus-\square+\square
\end{aligned}
$$

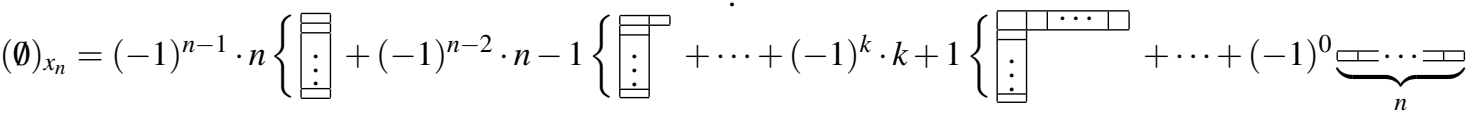

We observe that the $x_{1}$ derivative of a Young diagram is given by all possible diagrams formed by adding one plaquette with the form $\square$. The $x_{2}$ derivative of a Young diagram is given by all possible diagrams formed by adding one plaquette with the form $-\boxminus, \square$.The $x_{3}$ derivative of a Young diagram is given by all possible diagrams formed by adding one plaquette with the form $⿴ 囗 ⿱ 一 一,,-\square, \square \square$. The $x_{4}$ derivative of a Young diagram is given by all possible diagrams formed by adding one plaquette with the form -目, 目, 一个, The $x_{n}$ derivative of a Young diagram is given by all possible diagrams

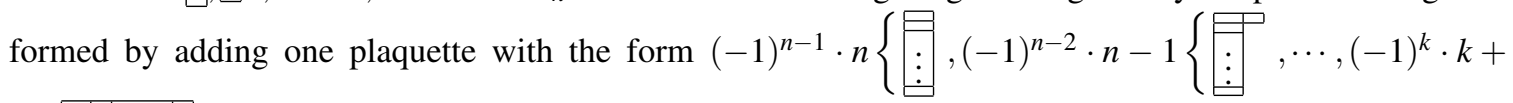

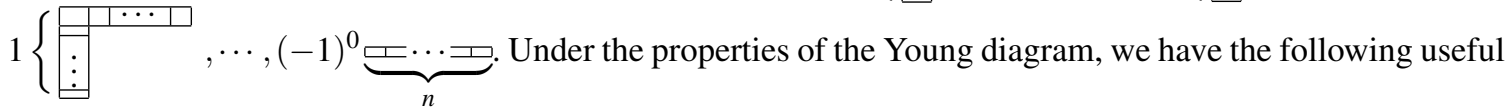
Lemmas.

Lemma 2.1. If $\tau=\widehat{|N-1|}=\emptyset$, from fourth-order Plücker relations, the following Young diagram identical equation hold

$$
\emptyset \times \boxminus-\square \times \boxminus+\square \times \boxminus=0 .
$$

Lemma 2.2. Under sixth-order Plücker relations, the following Young diagram identical equation hold

$$
\emptyset \times \varpi-\square \times \square+\square \times \square=0 .
$$

Lemma 2.3. Under sixth-order Plücker relations, the following Young diagram identical equation hold

$$
\emptyset \times \square-\square \times \square+\square \times \boxminus=0 .
$$

Lemma 2.4. The Young diagram identical equation hood

$$
\emptyset \times \boxminus-\square \times \boxminus+\square \times \boxminus=0 .
$$

Proof. We can compute derivatives of both sides of the fifth-order Plücker relations

$$
\emptyset \times \square-\square \times \square+\square \times \boxminus=0,
$$

with respect to the variables $x$ as follows:

$$
(\emptyset \times \boxminus-\square \times \square \square+\square \times \boxminus)_{\square}=0,
$$

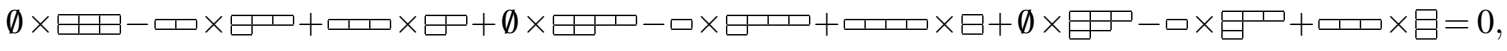
thus we obtain Lemma 2.4 by using Lemma 2.2 and Lemma 2.3 .

Lemma 2.5. The Young diagram identical equation

$$
\emptyset \times \boxminus-\boxminus \times \boxminus+\boxminus \times \boxminus=0,
$$

can be obtained by using diagonal symmetry transformation: 
to the sixth-order plücker relations in Lemma 2.2.

Proof.

$$
\begin{aligned}
& \emptyset \times \boxminus-\boxminus \times \boxminus+\boxminus \times \boxminus \\
& =|\widehat{N-1}||\widehat{N-4}, N-1, N, N+1|-|\widehat{N-3}, N-1, N||\widehat{N-4}, N-2, N-1, N+1| \\
& +|\widehat{N-3}, N-1, N+1||\widehat{N-4}, N-2, N-1, N| \\
& =(-1)^{2}|\widehat{N-4}, N-1, N-3, N-2||\widehat{N-4}, N-1, N, N+1| \\
& -(-1)|\widehat{N-4}, N-1, N-3, N|(-1)|\widehat{N-4}, N-1, N-2, N+1| \\
& +(-1)|\widehat{N-4}, N-1, N-3, N+1|(-1)|\widehat{N-4}, N-1, N-2, N| \\
& =|\widehat{N-4}, N-1, N-3, N-2||\widehat{N-4}, N-1, N, N+1| \\
& -|\widehat{N-4}, N-1, N-3, N||\widehat{N-4}, N-1, N-2, N+1| \\
& +|\widehat{N-4}, N-1, N-3, N+1||\widehat{N-4}, N-1, N-2, N| \\
& =0
\end{aligned}
$$

Lemma 2.6. The Young diagram identical equation

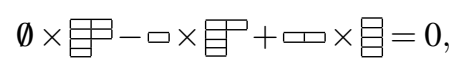

can be obtained by using diagonal symmetry transformation:

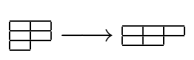

to the sixth-order plücker relations in Lemma 2.3.

Proof. We can compute derivatives of both sides of the fourth-order Plücker relations (2.6) with respect to the variables $x$ as follows:

$$
\begin{gathered}
(\emptyset \times \boxminus-\square \times \boxminus+\square \times \boxminus)_{\square}=0, \\
(\emptyset)_{\square} \times \boxminus+\emptyset \times(\boxminus)_{\square}(\square)_{\square} \times \boxminus-\square \times(\boxminus)_{\square}+(\square)_{\square} \times \boxminus+\square \times(\boxminus)_{\square}=0, \\
\emptyset \times \boxminus-\square \times \square \square+\square \times \boxminus+\emptyset \times \boxminus-\square \times \boxminus+\square \times \boxminus=0 .
\end{gathered}
$$

Thus we obtain

$$
\emptyset \times \boxminus-\square \times \boxminus+\varpi \times \boxminus=0
$$

by using the fifth-order Plücker relations (2.10)

We can compute derivatives of both sides of the above expression, with respect to the variables $x$ as follows:

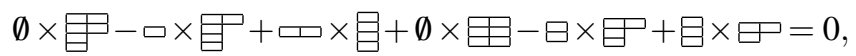

thus we obtain Lemma 2.6 by using Lemma 2.5 . 

hold

Character 1 Under the conditions $\sum_{j=1}^{N} a_{i j} \phi_{j}=\phi_{i x x}$, the following Young diagram identical equation

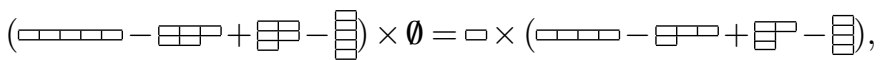

$$
\begin{aligned}
& \emptyset \times(\square-\text { 目 })=\square \times(\square-日), \\
& \emptyset \times(\square-\boxminus+2 \boxminus-日+\text { 目 })=(\square-日)^{2}, \\
& \emptyset \times(\square+2 \boxminus \square+\boxminus-\boxminus-2 \text { 四 }- \text { 目 })=(\square-\boxminus) \times(\square+2 \boxminus+\text { 目 })
\end{aligned}
$$

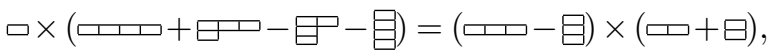

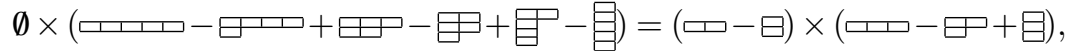

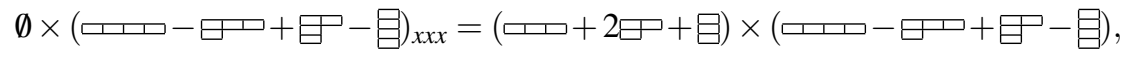

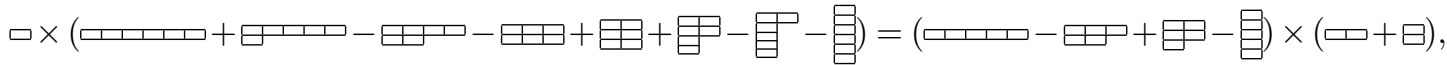

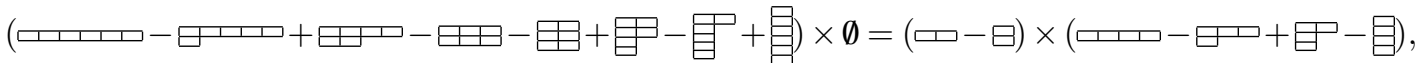

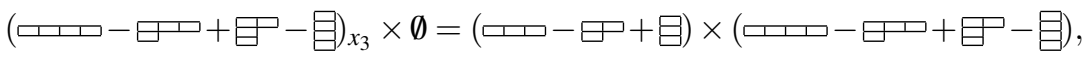

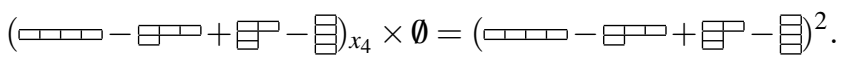

Proof. Under the properties of the Young diagram and the conditions $\sum_{j=1}^{N} a_{i j} \phi_{j}=\phi_{i x x}$, we can compute derivatives of the Young diagram $\tau=\emptyset$ with respect to the variables $x_{2}, x_{4}$ as follows

$$
\begin{aligned}
& (\emptyset)_{x_{2}}=\square-\boxminus=\sum_{i=1}^{N} a_{i i} \emptyset, \\
& (\emptyset)_{x_{4}}=\square-\boxminus+\boxminus-\text { 目 }=\sum_{i=1, j=1}^{N} a_{i j} a_{j i} \emptyset, \\
& (\emptyset)_{x_{1} x_{4}}=\square-\varpi+\boxminus-\boxminus=\sum_{i=1, j=1}^{N} a_{i j} a_{j i} \emptyset_{x_{1}}=\sum_{i=1, j=1}^{N} a_{i j} a_{j i} \bullet
\end{aligned}
$$

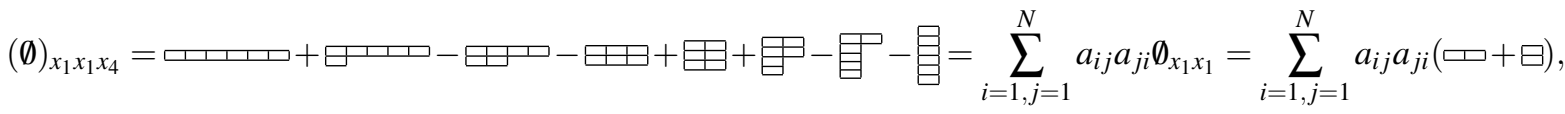

$$
\begin{aligned}
& (\emptyset)_{x_{2} x_{4}}=\square \text { - } \\
& (\emptyset)_{x_{2} x_{2}}=\square-\square+2 \boxminus-\boxminus+\text { 目 }=\left(\sum_{i=1}^{N} a_{i i}\right)^{2} \emptyset,
\end{aligned}
$$

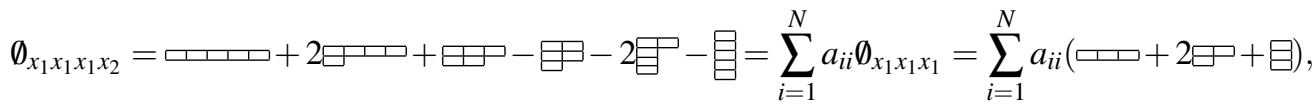

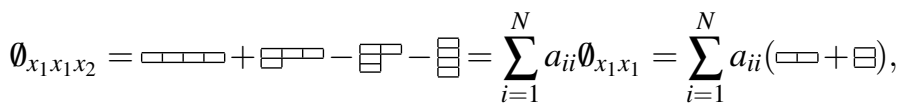

$$
\begin{aligned}
& \emptyset_{x_{1} x_{2}}=\square \text { 目 }=\sum_{i=1}^{N} a_{i i} \emptyset_{x_{1}}=\sum_{i=1}^{N} a_{i i} \sqsubset \text {, }
\end{aligned}
$$

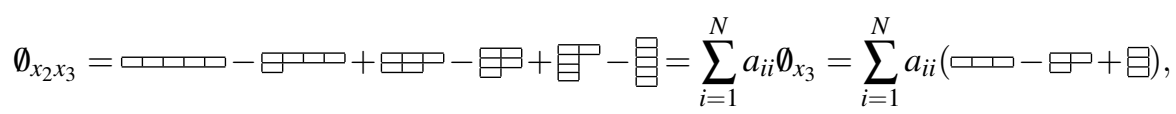

thus we obtain Character 1. 

hold

Character 2 Under the conditions $\sum_{j=1}^{N} a_{i j} \phi_{j}=\phi_{i x x x x}$, the following Young diagram identical equation

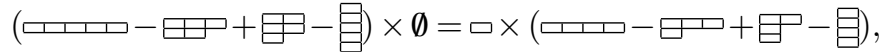

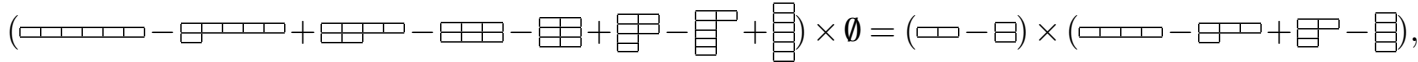

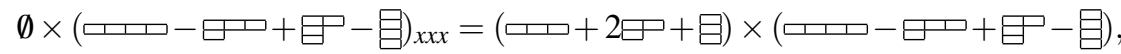

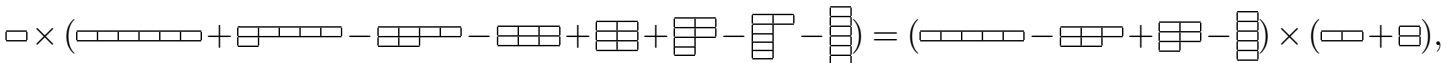

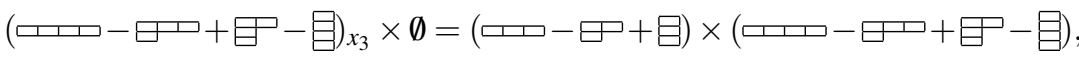

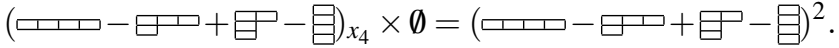

Proof. Under the properties of the Young diagram and the conditions $\sum_{j=1}^{N} a_{i j} \phi_{j}=\phi_{i x x x x}$, we can compute derivatives of the Young diagram $\tau=\emptyset$ with respect to the variables $x_{4}$ as follows

$$
\begin{aligned}
& (\emptyset)_{x_{4}}=\square-\square+\boxminus-\boxminus=\sum_{i=1}^{N} a_{i i} \emptyset, \\
& (\emptyset)_{x_{1} x_{4}}=\text { ロ }
\end{aligned}
$$

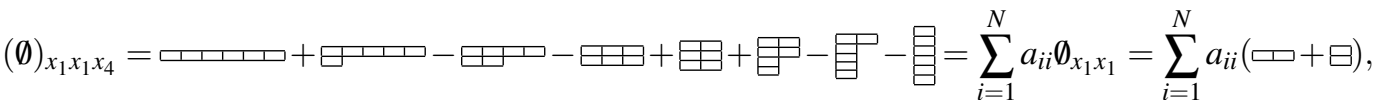

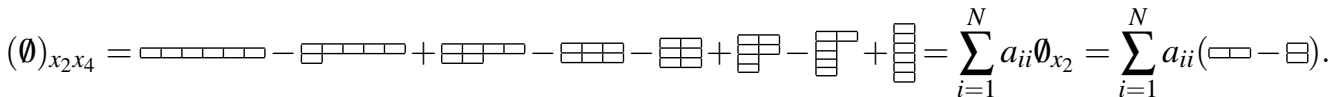

thus we obtain Character 2.

We give three generalized linear differential conditions for Wronskian solution of Eq.(1.2) in the next part.

Theorem 2.1. Assuming that $\phi_{i}(x, y, t) 1 \leq i \leq N$ has continuous derivative up to any order, and satisfies the following linear differential conditions

$$
\left\{\begin{array}{l}
\phi_{i y}=-2 \phi_{i x x x}+l \phi_{i x x x x} \\
\phi_{i t}=s \phi_{i x x x x}-4 \phi_{i x x x x x} \\
\sum_{j=1}^{N} a_{i j} \phi_{j}=\phi_{i x x x x}
\end{array}\right.
$$

then the Wronskian determinant $\tau$ defined by(2.1) is the solution of Eq(1.2). 
Proof. Under the properties of the Young diagram and the conditions(2.13). We can compute various derivatives of the Young diagram $\tau=\emptyset$ with respect to the variables $\mathrm{x}, \mathrm{y}, \mathrm{t}$.

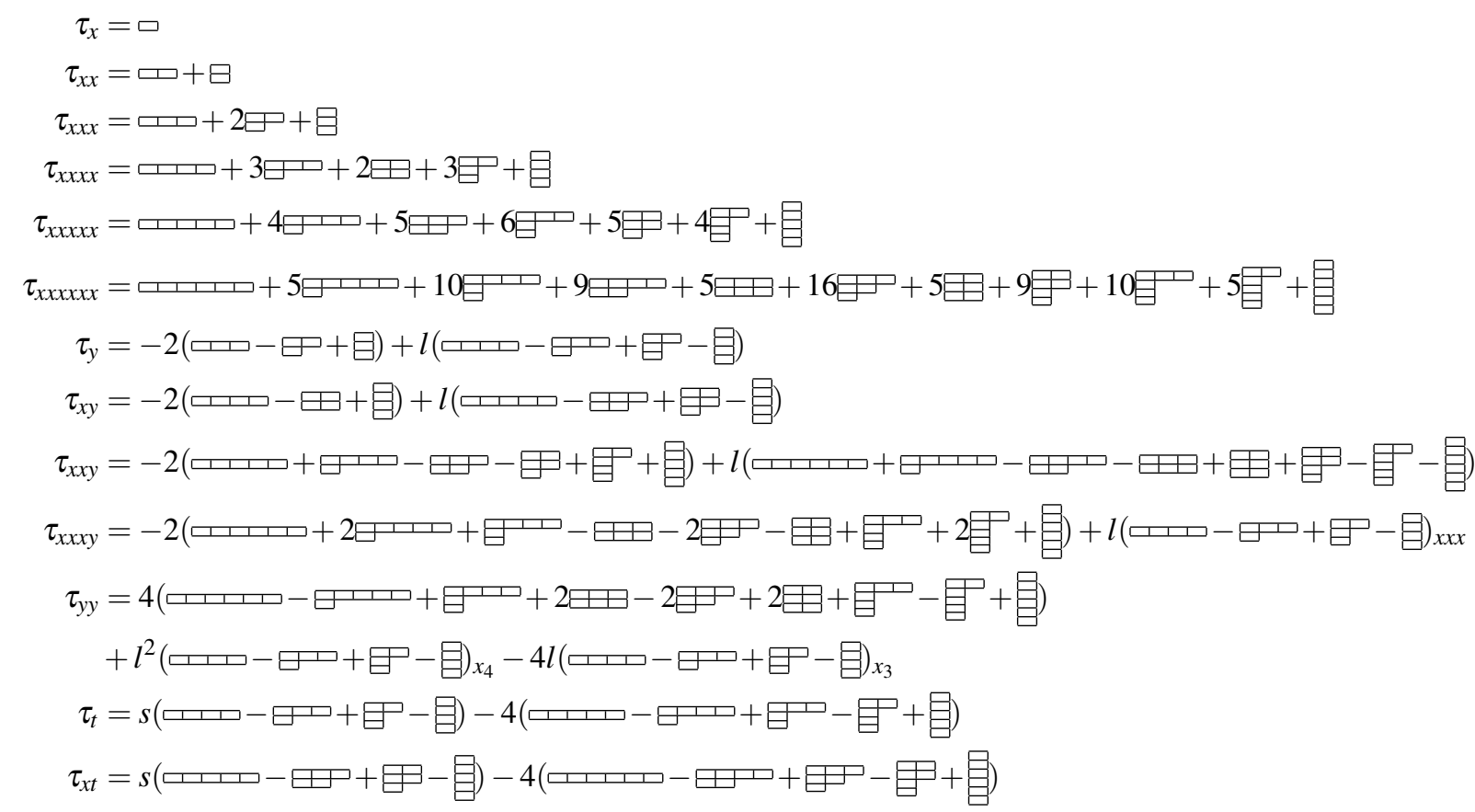

Substituting the above derivatives of $\tau$ into Eq.(1.2), from Lemma 2.2, 2.3, 2.5, 2.6 and Character 2 we obtain

$$
\begin{aligned}
& \left(D_{x}^{6}-5 D_{x}^{3} D_{y}-5 D_{y}^{2}+9 D_{x} D_{t}\right) \tau \tau \\
& =2\left[\left(\tau_{x x x x x x}-5 \tau_{x x x y}-5 \tau_{y y}+9 \tau_{x t}\right) \tau+\left(15 \tau_{x x y}-9 \tau_{t}-6 \tau_{x x x x x}\right) \tau_{x}-\left(10 \tau_{x x x} \tau_{x x x}-5 \tau_{x x x} \tau_{y}-5 \tau_{y}^{2}\right)\right. \\
& \left.+\left(15 \tau_{x x x x} \tau_{x x}-15 \tau_{x x} \tau_{x y}\right)\right]
\end{aligned}
$$

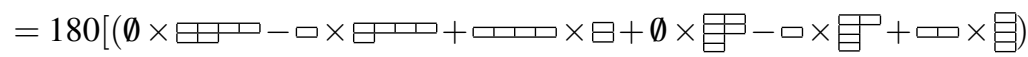

$$
\begin{aligned}
& -180(\emptyset \times \sqcap-\square \times \boxminus+\square \times \boxminus+\emptyset \times \boxminus-\boxminus \times \boxminus+\boxminus \times \boxminus)] \\
& =0 \text {. }
\end{aligned}
$$

Theorem 2.2. Assuming that $\phi_{i}(x, y, t) 1 \leq i \leq N$ has continuous derivative up to any order, and satisfies the following linear differential conditions

$$
\left\{\begin{array}{l}
\phi_{i y}=l \phi_{i x}+r \phi_{i x x}+4 \phi_{i x x x}+s \phi_{i x x x x}, \\
\phi_{i t}=\frac{5}{9} l^{2} \phi_{i x}+\alpha \phi_{i x x}+\frac{20}{3} l \phi_{i x x x}+\beta \phi_{i x x x x}+16 \phi_{i x x x x x}, \\
\sum_{j=1}^{N} a_{i j} \phi_{j}=\phi_{i x x}
\end{array}\right.
$$

then the Wronskian determinant $\tau$ defined by(2.1) is the solution of $E q(1.2)$.

Proof. Under the properties of the Young diagram and the conditions (2.14). In a similar with Theorem 2.1 , we can compute various derivatives of the Young diagram $\tau=\emptyset$ with respect to the variables $\mathrm{x}, \mathrm{y}, \mathrm{t}$. Substituting the derivatives of $\tau$ into Eq.(1.2), from Lemma 2.1, 2.2, 2.3, 2.4, 2.5, 2.6 and Character 1 we 
obtain

$$
\begin{aligned}
& \left(D_{x}^{6}-5 D_{x}^{3} D_{y}-5 D_{y}^{2}+9 D_{x} D_{t}\right) \tau \tau \\
& =2\left[\left(\tau_{x x x x x x}-5 \tau_{x x x y}-5 \tau_{y y}+9 \tau_{x t}\right) \tau+\left(15 \tau_{x x y}-9 \tau_{t}-6 \tau_{x x x x x}\right) \tau_{x}-\left(10 \tau_{x x x} \tau_{x x x}-5 \tau_{x x x} \tau_{y}-5 \tau_{y}^{2}\right)\right. \\
& \left.+\left(15 \tau_{x x x x} \tau_{x x}-15 \tau_{x x} \tau_{x y}\right)\right]
\end{aligned}
$$

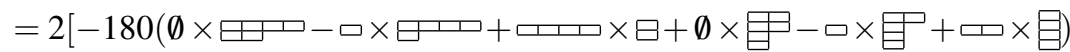

$$
\begin{aligned}
& -180(\emptyset \times \boxminus-\square \times \boxminus++\square \times \boxminus+\emptyset \times \boxminus-\boxminus \times \boxminus+\boxminus \times \square) \\
& +360(\emptyset \times \boxminus-\square \times \boxminus+\square+\square \times)]-120 l[\emptyset \times \boxminus-\square \times \square+\square \times \boxminus] \\
& =0 \text {. }
\end{aligned}
$$

Theorem 2.3. Assuming that $\phi_{i}(x, y, t) 1 \leq i \leq N$ has continuous derivative up to any order, and satisfies the following linear differential conditions

$$
\left\{\begin{array}{l}
\phi_{i y}=l \phi_{i x}+r \phi_{i x x}-2 \phi_{i x x x}+s \phi_{i x x x x} \\
\phi_{i t}=\frac{5}{9} l^{2} \phi_{i x}+\alpha \phi_{i x x}+\beta \phi_{i x x x x}-4 \phi_{i x x x x x} \\
\sum_{j=1}^{N} a_{i j} \phi_{j}=\phi_{i x x}
\end{array}\right.
$$

then the Wronskian determinant $\tau$ defined by(2.1) is the solution of Eq(1.2).

Proof. Under the properties of the Young diagram and the conditions (2.15). In a similar with Theorem 2.1 , we can compute various derivatives of the Young diagram $\tau=\emptyset$ with respect to the variables $\mathrm{x}, \mathrm{y}, \mathrm{t}$. Substituting the derivatives of $\tau$ into Eq.(1.2), from Lemma 2.1, 2.2, 2.3, 2.5, 2.6 and Character 1 we obtain

$$
\begin{aligned}
& \left(D_{x}^{6}-5 D_{x}^{3} D_{y}-5 D_{y}^{2}+9 D_{x} D_{t}\right) \tau \tau \\
& =2\left[\left(\tau_{x x x x x}-5 \tau_{x x x y}-5 \tau_{y y}+9 \tau_{x t}\right) \tau+\left(15 \tau_{x x y}-9 \tau_{t}-6 \tau_{x x x x x}\right) \tau_{x}-\left(10 \tau_{x x x} \tau_{x x x}-5 \tau_{x x x} \tau_{y}-5 \tau_{y}^{2}\right)\right. \\
& \left.+\left(15 \tau_{x x x x} \tau_{x x}-15 \tau_{x x} \tau_{x y}\right)\right]
\end{aligned}
$$

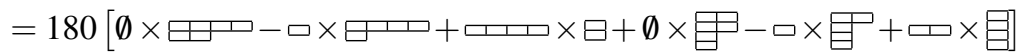

$$
\begin{aligned}
& -180[\emptyset \times \boxminus-\square \times \boxminus+\square \times \boxminus+\emptyset \times \boxminus-\boxminus \times \boxminus+\boxminus \times \boxminus] \\
& -120 l[\emptyset \times \boxminus-\square \times \boxminus+\square \times \boxminus] \\
& =0 \text {. }
\end{aligned}
$$

\section{Wronskian solutions to isospectral BKP equation}

\subsection{Wronskian solutions to isospectral BKP equation based on Wronskian condition (2.13)}

The Wronskian condition (2.13) can be written as the matrix form:

$$
A \Phi=\Phi_{x x x x}, \Phi_{y}=-2 \Phi_{x x x}+l \Phi_{x x x x}, \Phi_{t}=s \Phi_{x x x x}-4 \Phi_{x x x x},
$$

where $A=\left(a_{i j}\right)$ and $\Phi=\left(\phi_{1}, \phi_{2}, \ldots, \phi_{N}\right)^{T}$. Thus we only have to solve (3.1) under the Jordan form of $A$. We can take Jordan matrix $\mathrm{J}$ and its blocks $J_{i}$ as follows:

$$
J=\left(\begin{array}{cccc}
J_{1} & & & \\
& J_{2} & & \\
& & \ddots & \\
& & & J_{M}
\end{array}\right)_{N \times N}, J_{i}=\left(\begin{array}{cccc}
\lambda_{i} & & & \\
1 & \lambda_{i} & & \\
& \ddots & \ddots & \\
& & 1 & \lambda_{i}
\end{array}\right)_{k_{i} \times k_{i}}\left(\sum_{i=1}^{M} k_{i}=N\right),
$$

where $\lambda_{i}$ are real constants. Then (3.1) are transformed into:

$$
J_{i} \Phi_{i}=\Phi_{i, x x x x}, \Phi_{i, y}=-2 \Phi_{i, x x x}+l \Phi_{i, x x x x}, \Phi_{i, t}=s \Phi_{i, x x x x}-4 \Phi_{i, x x x x x}(1 \leq i \leq M)
$$


where $\Phi_{i}=\left(\phi_{i, 1}, \phi_{i, 2}, \ldots, \phi_{i, k_{i}}\right)^{T}$. Then

$$
u=2\left(\ln W r\left(\Phi_{1}^{T}, \Phi_{2}^{T}, \ldots, \Phi_{M}^{T}\right)\right)_{x x}
$$

is called the $\mathrm{N}$-Wronskian solution of $\left(k_{1}, k_{2}, \ldots, k_{M}\right)$-order to Eq.(1.1).

In this section, we consider different combinations of the $\Phi_{i}$ in Wronskian, various types of Wronskian solutions are obtained.

(i) Let $\lambda_{i}=0$, solving Eq.(3.2) obtain that

$$
\phi_{i, 1}=C_{4} y-\frac{1}{12} C_{4} x^{3}+\frac{1}{2} C_{1} x^{2}+C_{2} x+C_{3},
$$

where $C_{1}, C_{2}, C_{3}, C_{4}, C_{5}, \ldots$ are arbitrary constants. Then a rational solution of $\left(k_{i}\right)$-order to (3.2) reads

$$
\Phi_{i\left(k_{i}\right)}=\left(\phi_{i, 1}, \phi_{i, 2}, \ldots, \phi_{i, k_{i}}\right)^{T},
$$

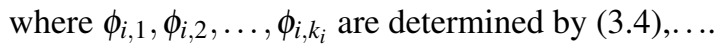

(ii) Let $\lambda_{i} \neq 0$, we can get a negaton $\left(\lambda_{i}>0\right)$ and positon $\left(\lambda_{i}<0\right)$ solution of $\left(k_{i}\right)$-order to (3.2):

$$
\Phi_{i\left(k_{i}\right)}=\left(\phi_{i, 1}\left(\lambda_{i}\right), \frac{1}{1 !} \partial_{\lambda_{i}} \phi_{i, 1}\left(\lambda_{i}\right), \ldots, \frac{1}{\left(k_{i}-1\right) !} \partial_{\lambda_{i}}^{k_{i}-1} \phi_{i, 1}\left(\lambda_{i}\right)\right)^{T},
$$

where $\phi_{i, 1}\left(\lambda_{i}\right)=e^{l \lambda_{i} y+s \lambda_{i} t}\left(c_{i, 1} e^{I \xi_{i}}+c_{i, 2} e^{\eta_{i}}+c_{i, 3} e^{-I \xi_{i}}+c_{i, 4} e^{-\eta_{i}}\right), \quad \xi_{i}=\lambda_{i}^{\frac{1}{4}} x+2 \lambda_{i}^{\frac{3}{4}} y-4 \lambda_{i}^{\frac{5}{4}} t+$ $\kappa_{i}, \eta_{i}=\lambda_{i}^{\frac{1}{4}} x-2 \lambda_{i}^{\frac{3}{4}} y-4 \lambda_{i}^{\frac{5}{4}} t+\varepsilon_{i}, l, s, c_{i, 1}, c_{i, 2}, c_{i, 3}, c_{i, 4}, \kappa_{i}, \varepsilon_{i}$ are arbitrary constants.

Case 1: If $\lambda_{i}>0, c_{i, 1}=c_{i, 3}=0$, the $(1,1, \ldots, 1)$-negaton solution become soliton solution.

$$
u=2\left(\ln W r\left(\phi_{1, \text { soliton }}, \phi_{2, \text { soliton }}, \ldots, \phi_{N, \text { soliton }}\right)\right)_{x x},
$$

where $\phi_{i, \text { soliton }}=e^{l \lambda_{i} y+s \lambda_{i} t}\left(c_{i, 2} e^{\eta_{i}}+(-1)^{i-1} c_{i, 4} e^{-\eta_{i}}\right), \eta_{i}=\lambda_{i}^{\frac{1}{4}} x-2 \lambda_{i}^{\frac{3}{4}} y-4 \lambda_{i}^{\frac{5}{4}} t+\varepsilon_{i}, l, s, c_{i, 2} c_{i, 4}>0, \varepsilon_{i}$ are arbitrary constants.

Case 2:If $\lambda_{i}>0, c_{i, 1}=c_{i, 3} \neq 0, c_{i, 2}=c_{i, 4}=0$, the $(1,1, \ldots, 1)$-negaton solution become trigonometric function solution.

$$
u=2\left(\ln W r\left(\phi_{1, \text { trigonometric }}, \phi_{2, \text { trigonometric }}, \ldots, \phi_{N, \text { trigonometric }}\right)\right)_{x x},
$$

where $\phi_{i, \text { trigonometric }}=e^{l \lambda_{i} y+s \lambda_{i} t}\left(c_{i, 1} e^{I \xi_{i}}+c_{i, 3} e^{-I \xi_{i}}\right), \xi_{i}=\lambda_{i}^{\frac{1}{4}} x+2 \lambda_{i}^{\frac{3}{4}} y-4 \lambda_{i}^{\frac{5}{4}} t+\kappa_{i}, l, s, \kappa_{i}$, are arbitrary constants.

Case 3:If $\lambda_{i}>0, c_{i, 1}=c_{i, 3} \neq 0$, the $(1,1, \ldots, 1)$-negaton solution become trigonometric-soliton solution.

$$
u=2\left(\ln W r\left(\phi_{1, \text { trigonometric-soliton }}, \phi_{2, \text { trigonometric-soliton }}, \ldots, \phi_{N, \text { trigonometric-soliton }}\right)\right)_{x x},
$$

where $\phi_{i, \text { trigonometric-soliton }}=e^{l \lambda_{i} y+s \lambda_{i} t}\left(c_{i, 1} e^{I \xi_{i}}+c_{i, 2} e^{\eta_{i}}+c_{i, 3} e^{-I \xi_{i}}+(-1)^{i-1} c_{i, 4} e^{-\eta_{i}}\right), \xi_{i}=\lambda_{i}^{\frac{1}{4}} x+2 \lambda_{i}^{\frac{3}{4}} y-$ $4 \lambda_{i}^{\frac{5}{4}} t+\kappa_{i}, \eta_{i}=\lambda_{i}^{\frac{1}{4}} x-2 \lambda_{i}^{\frac{3}{4}} y-4 \lambda_{i}^{\frac{5}{4}} t+\varepsilon_{i}, l, s, c_{i, 2} c_{i, 4}>0, \kappa_{i}, \varepsilon_{i}$ are arbitrary constants.

Case 4:If $\lambda_{i}>0, c_{i, 1} \neq c_{i, 3}$, the $(1,1, \ldots, 1)$-negaton solution become plural-soliton solution.

$$
u=2\left(\ln W r\left(\phi_{1}, \phi_{2}, \ldots, \phi_{N}\right)\right)_{x x},
$$

where $\phi_{i}=e^{l \lambda_{i} y+s \lambda_{i} t}\left(c_{i, 1} e^{I \xi_{i}}+c_{i, 2} e^{\eta_{i}}+c_{i, 3} e^{-I \xi_{i}}+(-1)^{i-1} c_{i, 4} e^{-\eta_{i}}\right), \quad \xi_{i}=\lambda_{i}^{\frac{1}{4}} x+2 \lambda_{i}^{\frac{3}{4}} y-4 \lambda_{i}^{\frac{5}{4}} t+\kappa_{i}, \eta_{i}=$ $\lambda_{i}^{\frac{1}{4}} x-2 \lambda_{i}^{\frac{3}{4}} y-4 \lambda_{i}^{\frac{5}{4}} t+\varepsilon_{i}, l, s, c_{i, 2} c_{i, 4}>0, \kappa_{i}, \varepsilon_{i}$ are arbitrary constants.

Case 5:If $\lambda_{i}>0, c_{i, 1}=c_{i, 3}=0$, the $(1,1, \ldots, 1)$-negaton solution become singular-soliton solution.

$$
u=2\left(\ln W r\left(\phi_{1}, \phi_{2}, \ldots, \phi_{N}\right)\right)_{x x},
$$

where $\phi_{i}=e^{l \lambda_{i} y+s \lambda_{i} t}\left(c_{i, 2} e^{\eta_{i}}+c_{i, 4} e^{-\eta_{i}}\right), \eta_{i}=\lambda_{i}^{\frac{1}{4}} x-2 \lambda_{i}^{\frac{3}{4}} y-4 \lambda_{i}^{\frac{5}{4}} t+\varepsilon_{i}, l, s, c_{i, 2} c_{i, 4}>0, \varepsilon_{i}$ are arbitrary constants. 
Case 6:If $\lambda_{i}>0, c_{i, 1}=c_{i, 3}=0, M \neq N$, the $\left(k_{1}, k_{2}, \ldots, k_{M}\right)$-order negaton solution become singularsoliton solution.

$$
\begin{aligned}
& u=2\left(\ln W r\left(\Phi_{1}^{T}, \Phi_{2}^{T}, \ldots, \Phi_{M}^{T}\right)\right)_{x x}, \\
& \Phi_{i\left(k_{i}\right)}=\left(\phi_{i, 1}\left(\lambda_{i}\right), \frac{1}{1 !} \partial_{\lambda_{i}} \phi_{i, 1}\left(\lambda_{i}\right), \ldots, \frac{1}{\left(k_{i}-1\right) !} \partial_{\lambda_{i}}^{k_{i}-1} \phi_{i, 1}\left(\lambda_{i}\right)\right)^{T},
\end{aligned}
$$

where $\phi_{i, 1}\left(\lambda_{i}\right)=e^{l \lambda_{i} y+s \lambda_{i} t}\left(c_{i, 2} e^{\eta_{i}}+c_{i, 4} e^{-\eta_{i}}\right), \eta_{i}=\lambda_{i}^{\frac{1}{4}} x-2 \lambda_{i}^{\frac{3}{4}} y-4 \lambda_{i}^{\frac{5}{4}} t+\varepsilon_{i}, l, s, c_{i, 2} c_{i, 4} \neq 0, \varepsilon_{i}$ are arbitrary constants.

Fig.1 displays the shape of the one soliton of (1)-order given by expression (3.7) and the TrigonometricSoliton solution of (1)-order given by expression (3.9). Fig.2 displays the shape of the Imaginary part, Real part and Length of Plural-Soliton solution of (1)-order given by expression (3.10).
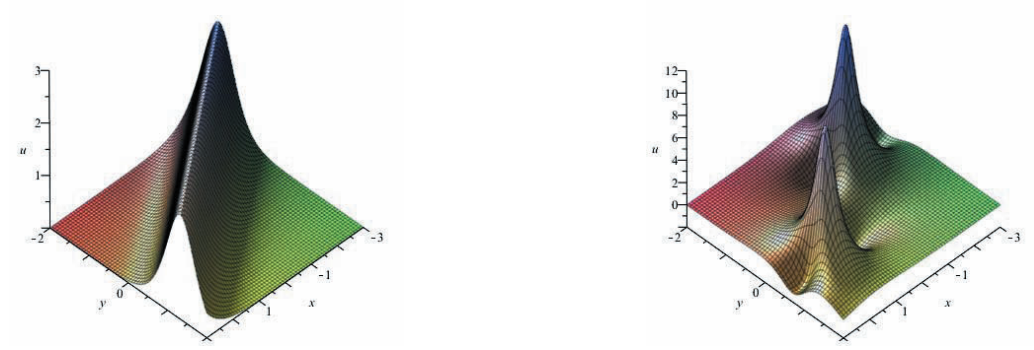

Fig 1. Taken at $\mathrm{t}=0$ to represent the different observations of the shape of one-soliton solution given by expression (3.7) with $c_{1,2}=1, c_{1,4}=2$, and Trigonometric-soliton solution given by expression (3.9) with $c_{1,1}=c_{1,3}=1, c_{1,2}=1, c_{1,4}=2$, where $l=1, s=-1, \lambda_{1}=1, \kappa_{1}=0, \varepsilon_{1}=0$
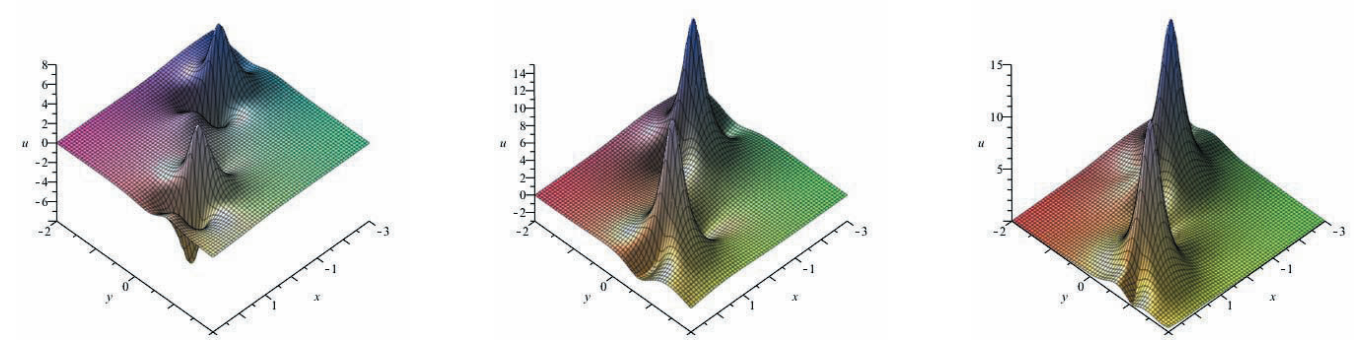

Fig 2. Taken at $\mathrm{t}=0$ to represent the different observations of the shape of the Imaginary part, Real part and Length of Plural-Soliton solution given by expression (3.10) with $c_{1,1}=1, c_{1,2}=2, c_{1,3}=3, c_{1,4}=4, l=1, s=-1, \lambda_{1}=1, \kappa_{1}=$ $0, \varepsilon_{1}=0$

Based on the soliton solutions, we give some figures to describe the propagation of the solitary waves. Fig. 3 and Fig.4 show the interaction of the two-soliton solutions of $(1,1)$-order and three-soliton solutions of $(1,1,1)$-order given by expression (3.7). Fig.5 and Fig.6 show the interaction of the two-Trigonometric-Soliton solutions of $(1,1)$-order given by expression (3.9).

Based on the singular-soliton solutions, we give some figures to describe the propagation of the singularsolitary waves. Fig.7 and Fig. 8 show the interaction of the three-singular-soliton solutions of $(1,1,1)$-order given by expression (3.11) and three-singular-soliton solutions of (3)-order given by expression (3.12). 

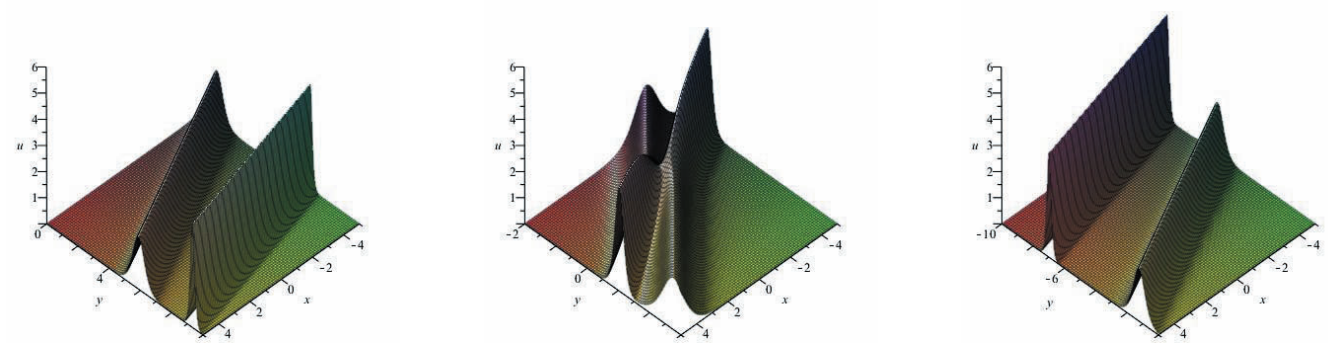

Fig 3. Taken at $\mathrm{t}=-2, \mathrm{t}=0, \mathrm{t}=2$ to represent the different observations of the fusion interaction of two-soliton solution given by expression (3.7) with $c_{i, 2}=1, c_{i, 4}=2, l=1, s=-1, \lambda_{1}=1, \lambda_{2}=4, \varepsilon_{1}=\varepsilon_{2}=0$
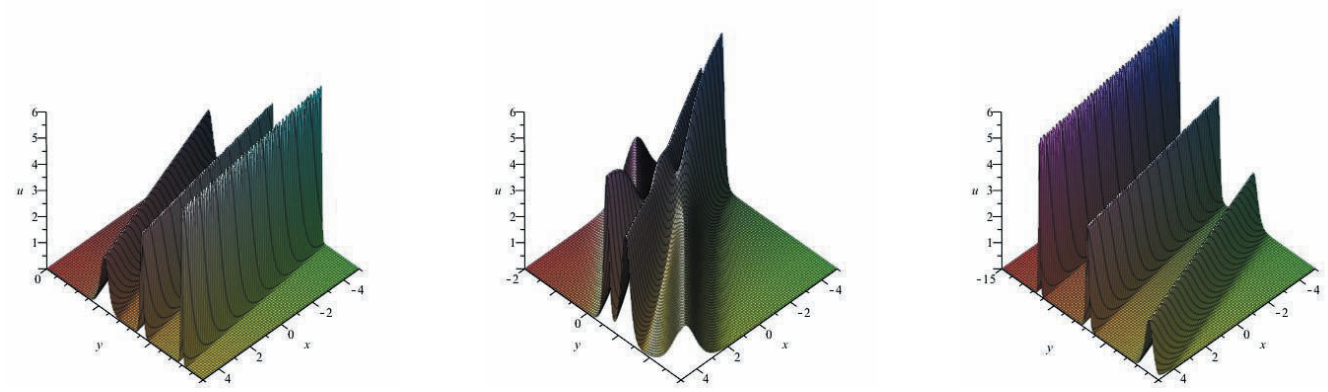

Fig 4. Taken at $\mathrm{t}=-2, \mathrm{t}=0, \mathrm{t}=2$ to represent the different observations of the fusion interaction of three-soliton solution given by expression (3.7) with $c_{i, 2}=1, c_{i, 4}=2, l=1, s=-1, \lambda_{1}=1, \lambda_{2}=4, \lambda_{3}=9, \varepsilon_{1}=\varepsilon_{2}=\varepsilon_{3}=0$
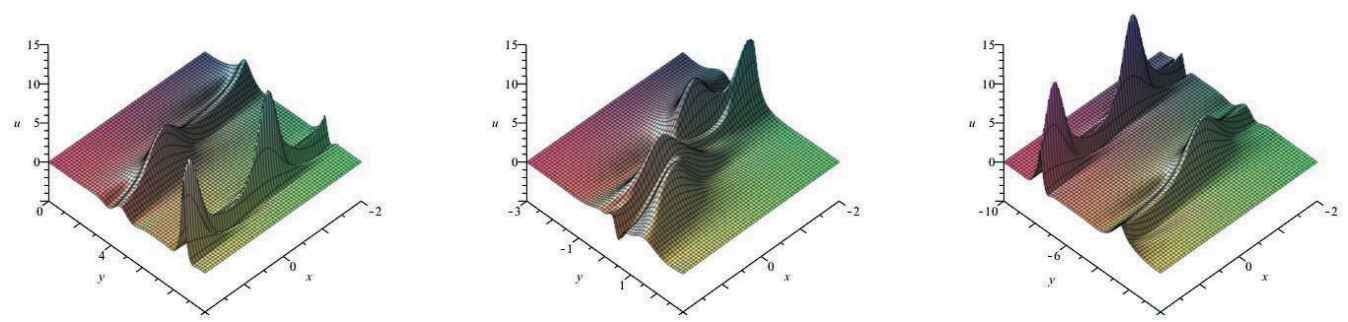

Fig 5. Taken at $\mathrm{t}=-2, \mathrm{t}=0, \mathrm{t}=2$ to represent the different observations of the fusion interaction of two-Trigonometric-Soliton solutions given by expression (3.9) with $c_{i, 1}=c_{i, 3}=1, c_{i, 2}=3, c_{i, 4}=4, l=1, s=-1, \lambda_{1}=1, \lambda_{2}=4, \kappa_{i}=\varepsilon_{i}=0$

\subsection{Wronskian solutions to isospectral BKP equation based on Wronskian condition (2.14) and}

Similarly with section 3.1, the Wronskian condition (2.14) can be written as the matrix form:

$$
\left\{\begin{array}{l}
A \Phi=\Phi_{x x} \\
\Phi_{y}=l \Phi_{x}+r \Phi_{x x}+4 \Phi_{x x x}+s \Phi_{x x x x} \\
\Phi_{t}=\frac{5}{9} l^{2} \Phi_{x}+\alpha \Phi_{x x}+\frac{20}{3} l \Phi_{x x x}+\beta \Phi_{x x x x}+16 \Phi_{x x x x x}
\end{array}\right.
$$

Co-published by Atlantis Press and Taylor \& Francis Copyright: the authors 

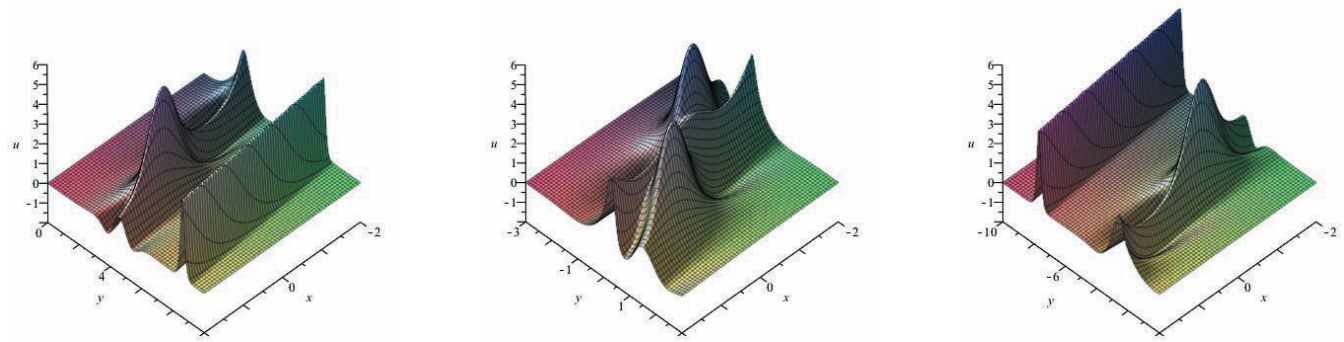

Fig 6. Taken at $\mathrm{t}=-2, \mathrm{t}=0, \mathrm{t}=2$ to represent the different observations of the fusion interaction of two-Trigonometric-Soliton solutions given by expression (3.9) with $c_{1,1}=c_{1,3}=1, c_{2,1}=c_{2,3}=0, c_{i, 2}=3, c_{i, 4}=4, l=1, s=-1, \lambda_{1}=1, \lambda_{2}=$ $4, \kappa_{i}=\varepsilon_{i}=0$
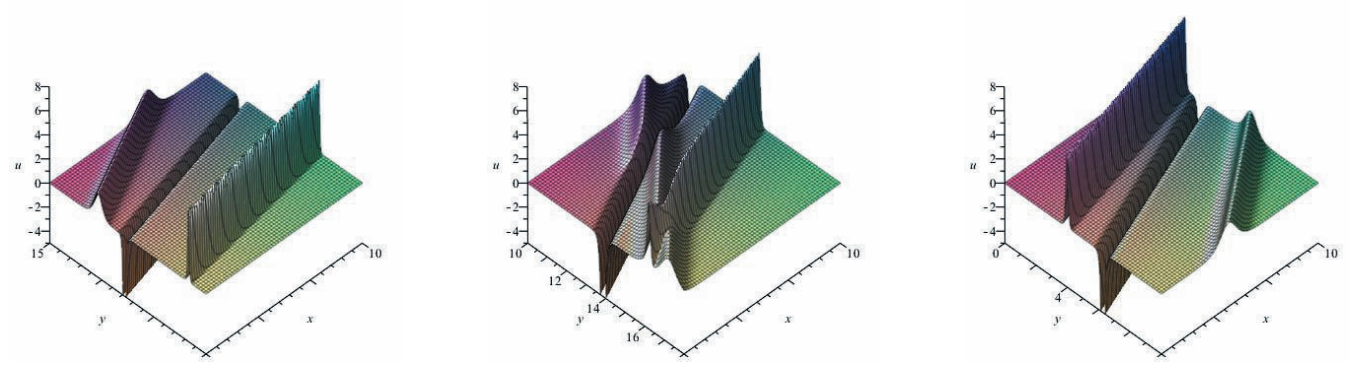

Fig 7. Taken at $\mathrm{t}=-4, \mathrm{t}=-2, \mathrm{t}=0$ to represent the different observations of the fusion interaction of three-singular-soliton solution given by expression (3.11) with $c_{i, 2}=3, c_{i, 4}=4, l=0, s=0, \lambda_{1}=1, \lambda_{2}=4, \lambda_{3}=9, \varepsilon_{1}=\varepsilon_{2}=\varepsilon_{3}=0$
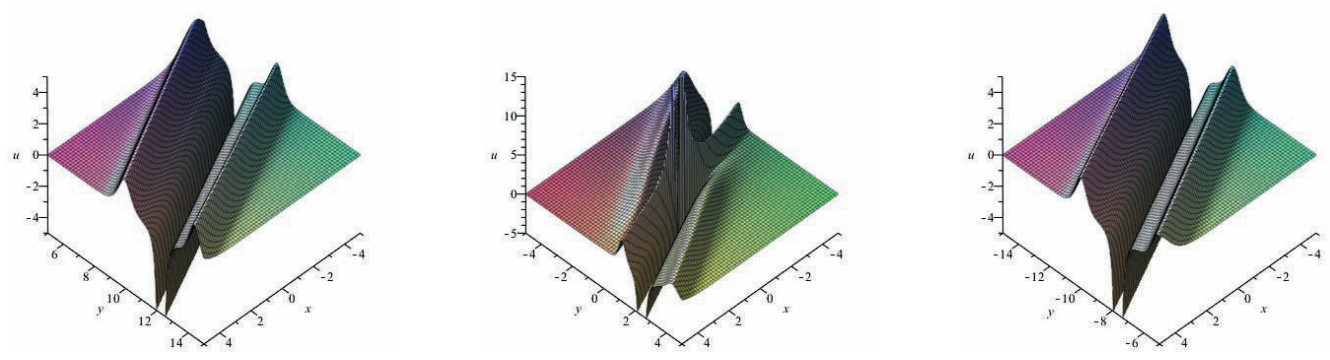

Fig 8 . Taken at $\mathrm{t}=-5, \mathrm{t}=0, \mathrm{t}=5$ to represent the different observations of the fusion interaction of three-singular-soliton solution given by expression (3.12) with $c_{1,2}=1, c_{1,4}=2, l=1, s=-1, \lambda_{1}=1, \varepsilon_{1}=0$

where $A=\left(a_{i j}\right)$ and $\Phi=\left(\phi_{1}, \phi_{2}, \ldots, \phi_{N}\right)^{T}$. Thus we only have to solve (3.14) under the Jordan form of A. Then (3.14) are transformed into:

$$
\left\{\begin{array}{l}
J_{i} \Phi_{i}=\Phi_{i, x x}, \\
\Phi_{i, y}=l \Phi_{i, x}+r \Phi_{i, x x}+4 \Phi_{i, x x x}+s \Phi_{i, x x x x} \\
\Phi_{i, t}=\frac{5}{9} l^{2} \Phi_{i, x}+\alpha \Phi_{i, x x}+\frac{20}{3} l \Phi_{i, x x x}+\beta \Phi_{i, x x x x}+16 \Phi_{i, x x x x x}(1 \leq i \leq M),
\end{array}\right.
$$

where $\Phi_{i}=\left(\phi_{i, 1}, \phi_{i, 2}, \ldots, \phi_{i, k_{i}}\right)^{T}$, Then

$$
u=2\left(\ln W r\left(\Phi_{1}^{T}, \Phi_{2}^{T}, \ldots, \Phi_{M}^{T}\right)\right)_{x x},
$$

Co-published by Atlantis Press and Taylor \& Francis Copyright: the authors 
is called the N-Wronskian solution of $\left(k_{1}, k_{2}, \ldots, k_{M}\right)$-order to Eq.(1.1).

Similarly, the Wronskian condition (2.15) can be written as the matrix form:

$$
\left\{\begin{array}{l}
A \Phi=\Phi_{x x} \\
\Phi_{y}=l \Phi_{x}+r \Phi_{x x}-2 \Phi_{x x x}+s \Phi_{x x x x} \\
\Phi_{t}=\frac{5}{9} l^{2} \Phi_{x}+\alpha \Phi_{x x}+\beta \Phi_{x x x x}-4 \Phi_{x x x x x}
\end{array}\right.
$$

where $A=\left(a_{i j}\right)$ and $\Phi=\left(\phi_{1}, \phi_{2}, \ldots, \phi_{N}\right)^{T}$. Thus we only have to solve (3.24) under the Jordan form of A. Then (3.24) are transformed into:

$$
\left\{\begin{array}{l}
J_{i} \Phi_{i}=\Phi_{i, x x}, \\
\Phi_{i, y}=l \Phi_{i, x}+r \Phi_{i, x x}-2 \Phi_{i, x x x}+s \Phi_{i, x x x x}, \\
\Phi_{i, t}=\frac{5}{9} l^{2} \Phi_{i, x}+\alpha \Phi_{i, x x}+\beta \Phi_{i, x x x x}-4 \Phi_{i, x x x x x}(1 \leq i \leq M),
\end{array}\right.
$$

where $\Phi_{i}=\left(\phi_{i, 1}, \phi_{i, 2}, \ldots, \phi_{i, k_{i}}\right)^{T}$, Then

$$
u=2\left(\ln W r\left(\Phi_{1}^{T}, \Phi_{2}^{T}, \ldots, \Phi_{M}^{T}\right)\right)_{x x},
$$

is called the $\mathrm{N}$-Wronskian solution of $\left(k_{1}, k_{2}, \ldots, k_{M}\right)$-order to Eq.(1.1).

In a similar, we give three generalized linear differential conditions for Grammian solution of Eq.(1.2) in the next part.

\section{Grammian solutions}

Let us now introduce the following Grammian determinant:

$$
\begin{gathered}
\tau=\operatorname{det}\left(a_{i j}\right), 1 \leq i, j \leq N, \\
a_{i j}=c_{i j}+\int^{x} f_{i} g_{j} d x,
\end{gathered}
$$

where $c_{i j}$ is constant.

Theorem 4.1. Assuming that $f_{i}(x, y, t), g_{j}(x, y, t) 1 \leq i, j \leq N$ has continuous derivative up to any order, and satisfies the following linear differential conditions

$$
\left\{\begin{array}{l}
f_{i y}=-2 f_{i x x x}+l f_{i x x x x}, g_{j y}=-2 g_{j x x x}-l g_{i x x x x} \\
f_{i t}=s f_{i x x x}-4 f_{i x x x x}, g_{j t}=-s g_{i x x x x}-4 g_{j x x x x} \\
f_{i x x x x}=a_{i} f_{i}, g_{j x x x}=b_{i} g_{j} \\
c_{i j}=0
\end{array}\right.
$$

then the Grammian determinant $\tau$ defined by Eq.(4.1) is the solution of Eq.(1.2).

Proof. Let us express the determinant $\tau$ by means of an $\mathrm{N}$-th order Pfaffian as

$$
\tau=\left(1,2, \ldots, N, N^{*}, \ldots, 2^{*}, 1^{*}\right),
$$

where $a_{i j}=\left(i, j^{*}\right)$, and $(i, j)=0,\left(i^{*}, j^{*}\right)=0$. In terms of these new entries and $\mathrm{Eq}(4.3)$, the derivatives of the entries $a_{i j}=\left(i, j^{*}\right)$ are given by

$$
\begin{gathered}
\frac{\partial}{\partial x} a_{i j}=f_{i} g_{j}=\left(d_{0}, d_{0}^{*}, i, j^{*}\right), \\
\frac{\partial}{\partial y} a_{i j}=-2\left[\left(d_{2}, d_{0}^{*}, i, j^{*}\right)-\left(d_{1}, d_{1}^{*}, i, j^{*}\right)+\left(d_{0}, d_{2}^{*}, i, j^{*}\right)\right] \\
+l\left[-\left(d_{3}, d_{0}^{*}, i, j^{*}\right)+\left(d_{2}, d_{1}^{*}, i, j^{*}\right)-\left(d_{1}, d_{2}^{*}, i, j^{*}\right)+\left(d_{0}, d_{3}^{*}, i, j^{*}\right)\right], \\
\frac{\partial}{\partial t} a_{i j}=s\left[-\left(d_{3}, d_{0}^{*}, i, j^{*}\right)+\left(d_{2}, d_{1}^{*}, i, j^{*}\right)-\left(d_{1}, d_{2}^{*}, i, j^{*}\right)+\left(d_{0}, d_{3}^{*}, i, j^{*}\right)\right] \\
-4\left[\left(d_{4}, d_{0}^{*}, i, j^{*}\right)-\left(d_{3}, d_{1}^{*}, i, j^{*}\right)+\left(d_{2}, d_{2}^{*}, i, j^{*}\right)-\left(d_{1}, d_{3}^{*}, i, j^{*}\right)+\left(d_{0}, d_{4}^{*}, i, j^{*}\right)\right],
\end{gathered}
$$

where

$$
\left\{\begin{array}{l}
\left(d_{n}, j^{*}\right)=\frac{\partial^{n}}{\partial x^{n}} g_{j},\left(d_{m}, d_{n}^{*}\right)=0 \\
\left(d_{n}^{*}, i\right)=\frac{\partial^{n}}{\partial x^{n}} f_{i},\left(d_{n}, i\right)=\left(d_{m}^{*}, i^{*}\right)=0 .
\end{array}\right.
$$


Then we can develop differential rules for Pfaffians, and compute various derivatives of the Grammian determinant with respect to variables $\mathrm{x}, \mathrm{y}$, and $\mathrm{t}$.

$$
\begin{gathered}
\frac{\partial \tau}{\partial y}=-2\left[\left(d_{2}, d_{0}^{*}, \bullet\right)-\left(d_{1}, d_{1}^{*}, \bullet\right)+\left(d_{0}, d_{2}^{*}, \bullet\right)\right]+l\left[-\left(d_{3}, d_{0}^{*}, \bullet\right)+\left(d_{2}, d_{1}^{*}, \bullet\right)-\left(d_{1}, d_{2}^{*}, \bullet\right)+\left(d_{0}, d_{3}^{*}, \bullet\right)\right], \\
\frac{\partial \tau}{\partial t}=s\left[-\left(d_{3}, d_{0}^{*}, \bullet\right)+\left(d_{2}, d_{1}^{*}, \bullet\right)-\left(d_{1}, d_{2}^{*}, \bullet\right)+\left(d_{0}, d_{3}^{*}, \bullet\right)\right]- \\
4\left[\left(d_{4}, d_{0}^{*}, \bullet\right)-\left(d_{3}, d_{1}^{*}, \bullet\right)+\left(d_{2}, d_{2}^{*}, \bullet\right)-\left(d_{1}, d_{3}^{*}, \bullet\right)+\left(d_{0}, d_{4}^{*}, \bullet\right)\right] .
\end{gathered}
$$

Where the abbreviated notation $\bullet$ denotes the list of indices $1,2, \ldots, N, N^{*}, \ldots, 2^{*}, 1^{*}$.

Substituting the above derivatives of $\tau$ into Eq.(1.2), from $f_{i x x x x}=a_{i} f_{i}, g_{j x x x x}=b_{i} g_{j}, c_{i j}=0$ we obtain

$$
\begin{aligned}
& \left(D_{x}^{6}-5 D_{x}^{3} D_{y}-5 D_{y}^{2}+9 D_{x} D_{t}\right) \tau \tau \\
& =2\left[\left(\tau_{x x x x x x}-5 \tau_{x x x y}-5 \tau_{y y}+9 \tau_{x t}\right) \tau+\left(15 \tau_{x x y}-9 \tau_{t}-6 \tau_{x x x x x}\right) \tau_{x}-\left(10 \tau_{x x x} \tau_{x x x}-5 \tau_{x x x} \tau_{y}-5 \tau_{y}^{2}\right)\right. \\
& \left.+\left(15 \tau_{x x x x} \tau_{x x}-15 \tau_{x x} \tau_{x y}\right)\right] \\
& =180\left[A_{1}+A_{2}\right]-180\left[B_{1}+B_{2}\right],
\end{aligned}
$$

where

$$
\begin{aligned}
& A_{1}=\left(d_{0}, d_{0}^{*}, d_{1}, d_{3}^{*}, \bullet\right)(\bullet)-\left(d_{0}, d_{0}^{*}, \bullet\right)\left(d_{1}, d_{3}^{*}, \bullet\right)+\left(d_{0}, d_{3}^{*}, \bullet\right)\left(d_{1}, d_{0}^{*}, \bullet\right)=0, \\
& A_{2}=\left(d_{0}, d_{0}^{*}, d_{3}, d_{1}^{*}, \bullet\right)(\bullet)-\left(d_{0}, d_{0}^{*}, \bullet\right)\left(d_{3}, d_{1}^{*}, \bullet\right)+\left(d_{3}, d_{0}^{*}, \bullet\right)\left(d_{0}, d_{1}^{*}, \bullet\right)=0, \\
& B_{1}=\left(d_{0}, d_{1}^{*}, d_{1}, d_{2}^{*}, \bullet\right)(\bullet)-\left(d_{0}, d_{1}^{*}, \bullet\right)\left(d_{1}, d_{2}^{*}, \bullet\right)+\left(d_{0}, d_{2}^{*}, \bullet\right)\left(d_{1}, d_{1}^{*}, \bullet\right)=0, \\
& B_{2}=\left(d_{1}, d_{0}^{*}, d_{2}, d_{1}^{*}, \bullet\right)(\bullet)-\left(d_{1}, d_{0}^{*}, \bullet\right)\left(d_{2}, d_{1}^{*}, \bullet\right)+\left(d_{2}, d_{0}^{*}, \bullet\right)\left(d_{1}, d_{1}^{*}, \bullet\right)=0 .
\end{aligned}
$$

It is easy to see that Eq.(4.9) is nothing but the Jacobi identity for determinants. Therefore, we show that $\tau$ is the solution of Eq.(1.2) under the linear differential conditions (4.3).

Theorem 4.2. Assuming that $f_{i}(x, y, t), g_{j}(x, y, t) 1 \leq i, j \leq N$ has continuous derivative up to any order, and satisfies the following linear differential conditions

$$
\left\{\begin{array}{l}
f_{i y}=l f_{i x}+r f_{i x x}+4 f_{i x x x}+s f_{i x x x x}, g_{j y}=l g_{j x}-r g_{j x x}+4 g_{j x x x}-s g_{j x x x} \\
f_{i t}=\frac{5}{9} l^{2} f_{i x}+\alpha f_{i x x}+\frac{20}{3} l f_{i x x x}+\beta f_{i x x x x}+16 f_{i x x x x} \\
g_{j t}=\frac{5}{9} l^{2} g_{j x}-\alpha g_{j x x}+\frac{20}{3} l g_{j x x x}-\beta g_{j x x x}+16 g_{j x x x x} \\
f_{i x x}=a_{i} f_{i}, g_{j x x}=b_{i} g_{j} \\
c_{i j}=0
\end{array}\right.
$$

then the Grammian determinant $\tau$ defined by Eq.(4.1) is the solution of Eq.(1.2).

Proof. In a similar with the Proof Theorem 4.1, we can compute various derivatives of the Grammian determinant with respect to variables $\mathrm{x}, \mathrm{y}$ and $\mathrm{t}$. Substituting the above derivatives of $\tau$ into Eq.(1.2), we obtain

$$
\begin{aligned}
& \left(D_{x}^{6}-5 D_{x}^{3} D_{y}-5 D_{y}^{2}+9 D_{x} D_{t}\right) \tau \tau \\
& =2\left[\left(\tau_{x x x x x x}-5 \tau_{x x x y}-5 \tau_{y y}+9 \tau_{x t}\right) \tau+\left(15 \tau_{x x y}-9 \tau_{t}-6 \tau_{x x x x x}\right) \tau_{x}-\left(10 \tau_{x x x} \tau_{x x x}-5 \tau_{x x x} \tau_{y}-5 \tau_{y}^{2}\right)\right. \\
& \left.+\left(15 \tau_{x x x x} \tau_{x x}-15 \tau_{x x} \tau_{x y}\right)\right] \\
& =-360\left[A_{1}+A_{2}\right]-360\left[B_{1}+B_{2}\right]+720 C-120 l D,
\end{aligned}
$$

where

$$
\begin{aligned}
A_{1} & =\left(d_{0}, d_{0}^{*}, d_{1}, d_{3}^{*}, \bullet\right)(\bullet)-\left(d_{0}, d_{0}^{*}, \bullet\right)\left(d_{1}, d_{3}^{*}, \bullet\right)+\left(d_{0}, d_{3}^{*}, \bullet\right)\left(d_{1}, d_{0}^{*}, \bullet\right)=0, \\
A_{2} & =\left(d_{0}, d_{0}^{*}, d_{3}, d_{1}^{*}, \bullet\right)(\bullet)-\left(d_{0}, d_{0}^{*}, \bullet\right)\left(d_{3}, d_{1}^{*}, \bullet\right)+\left(d_{3}, d_{0}^{*}, \bullet\right)\left(d_{0}, d_{1}^{*}, \bullet\right)=0, \\
B_{1} & =\left(d_{0}, d_{1}^{*}, d_{1}, d_{2}^{*}, \bullet\right)(\bullet)-\left(d_{0}, d_{1}^{*}, \bullet\right)\left(d_{1}, d_{2}^{*}, \bullet\right)+\left(d_{0}, d_{2}^{*}, \bullet\right)\left(d_{1}, d_{1}^{*}, \bullet\right)=0, \\
B_{2} & =\left(d_{1}, d_{0}^{*}, d_{2}, d_{1}^{*}, \bullet\right)(\bullet)-\left(d_{1}, d_{0}^{*}, \bullet\right)\left(d_{2}, d_{1}^{*}, \bullet\right)+\left(d_{2}, d_{0}^{*}, \bullet\right)\left(d_{1}, d_{1}^{*}, \bullet\right)=0, \\
C & =\left(d_{0}, d_{0}^{*}, d_{2}, d_{2}^{*}, \bullet\right)(\bullet)-\left(d_{0}, d_{0}^{*}, \bullet\right)\left(d_{2}, d_{2}^{*}, \bullet\right)+\left(d_{2}, d_{0}^{*}, \bullet\right)\left(d_{0}, d_{2}^{*}, \bullet\right)=0, \\
D & =\left(d_{0}, d_{0}^{*}, d_{1}, d_{1}^{*}, \bullet\right)(\bullet)-\left(d_{0}, d_{0}^{*}, \bullet\right)\left(d_{1}, d_{1}^{*}, \bullet\right)+\left(d_{0}, d_{1}^{*}, \bullet\right)\left(d_{1}, d_{0}^{*}, \bullet\right)=0 .
\end{aligned}
$$

It is easy to see that Eq.(4.11) is nothing but the Jacobi identity for determinants. Therefore, we show that $\tau$ is the solution of Eq.(1.2) under the linear differential conditions (4.10). 
Theorem 4.3. Assuming that $f_{i}(x, y, t), g_{j}(x, y, t) 1 \leq i, j \leq N$ has continuous derivative up to any order, and satisfies the following linear differential conditions

$$
\left\{\begin{array}{l}
f_{i y}=l f_{i x}+r f_{i x x}-2 f_{i x x x}+s f_{i x x x x}, g_{j y}=l g_{j x}-r g_{j x x}-2 g_{j x x x}-s g_{j x x x x} \\
f_{i t}=\frac{5}{9} l^{2} f_{i x}+\alpha f_{i x x}+\beta f_{i x x x x}-4 f_{i x x x x} \\
g_{j t}=\frac{5}{9} l^{2} g_{j x}-\alpha g_{j x x}-\beta g_{j x x x x}-4 g_{j x x x x} \\
f_{i x x}=a_{i} f_{i}, g_{j x x}=b_{i} g_{j} \\
c_{i j}=0
\end{array}\right.
$$

then the Grammian determinant $\tau$ defined by Eq.(4.1) is the solution of Eq.(1.2).

Proof. In a similar with the Proof Theorem 4.1, we can compute various derivatives of the Grammian determinant with respect to variables $\mathrm{x}, \mathrm{y}$ and $\mathrm{t}$. Substituting the above derivatives of $\tau$ into Eq.(1.2), we obtain

$$
\begin{aligned}
& \left(D_{x}^{6}-5 D_{x}^{3} D_{y}-5 D_{y}^{2}+9 D_{x} D_{t}\right) \tau \tau \\
& =2\left[\left(\tau_{x x x x x x}-5 \tau_{x x x y}-5 \tau_{y y}+9 \tau_{x t}\right) \tau+\left(15 \tau_{x x y}-9 \tau_{t}-6 \tau_{x x x x x}\right) \tau_{x}-\left(10 \tau_{x x x} \tau_{x x x}-5 \tau_{x x x} \tau_{y}-5 \tau_{y}^{2}\right)\right. \\
& \left.+\left(15 \tau_{x x x x} \tau_{x x}-15 \tau_{x x} \tau_{x y}\right)\right] \\
& =180\left[A_{1}+A_{2}\right]-180\left[B_{1}+B_{2}\right]-120 l D,
\end{aligned}
$$

where

$$
\begin{aligned}
A_{1} & =\left(d_{0}, d_{0}^{*}, d_{1}, d_{3}^{*}, \bullet\right)(\bullet)-\left(d_{0}, d_{0}^{*}, \bullet\right)\left(d_{1}, d_{3}^{*}, \bullet\right)+\left(d_{0}, d_{3}^{*}, \bullet\right)\left(d_{1}, d_{0}^{*}, \bullet\right)=0, \\
A_{2} & =\left(d_{0}, d_{0}^{*}, d_{3}, d_{1}^{*}, \bullet\right)(\bullet)-\left(d_{0}, d_{0}^{*}, \bullet\right)\left(d_{3}, d_{1}^{*}, \bullet\right)+\left(d_{3}, d_{0}^{*}, \bullet\right)\left(d_{0}, d_{1}^{*}, \bullet\right)=0, \\
B_{1} & =\left(d_{0}, d_{1}^{*}, d_{1}, d_{2}^{*}, \bullet\right)(\bullet)-\left(d_{0}, d_{1}^{*}, \bullet\right)\left(d_{1}, d_{2}^{*}, \bullet\right)+\left(d_{0}, d_{2}^{*}, \bullet\right)\left(d_{1}, d_{1}^{*}, \bullet\right)=0, \\
B_{2} & =\left(d_{1}, d_{0}^{*}, d_{2}, d_{1}^{*}, \bullet\right)(\bullet)-\left(d_{1}, d_{0}^{*}, \bullet\right)\left(d_{2}, d_{1}^{*}, \bullet\right)+\left(d_{2}, d_{0}^{*}, \bullet\right)\left(d_{1}, d_{1}^{*}, \bullet\right)=0, \\
D & =\left(d_{0}, d_{0}^{*}, d_{1}, d_{1}^{*}, \bullet\right)(\bullet)-\left(d_{0}, d_{0}^{*}, \bullet\right)\left(d_{1}, d_{1}^{*}, \bullet\right)+\left(d_{0}, d_{1}^{*}, \bullet\right)\left(d_{1}, d_{0}^{*}, \bullet\right)=0 .
\end{aligned}
$$

It is easy to see that Eq.(4.13) is nothing but the Jacobi identity for determinants. Therefore, we show that $\tau$ is the solution of Eq.(1.2) under the linear differential conditions (4.12).

\section{Conclusion}

In summary, we have established generalized Wronskian and Grammian solutions for the isospectral B-type Kadomtsev-Petviashvili (isospectral BKP) equation (1.1). Our results show that Eq. (1.1) not only has Pfaffian solutions but also has Grammian determinant solutions and Wronskian determinant solutions. This property is completely different from that of the KP equation, which only has Grammian solutions and Wronskian determinant solutions, and from that of the BKP equation, which only has Pfaffian solutions. By considering the different combinations of the entries in Wronskian, we obtain various types of Wronskian solutions.

Generally speaking, the KP hierarchy which only has Grammian and Wronskian determinant solutions, and from that of the BKP hierarchy, which only has Pfaffian solutions. But Tang etal [26] have established Grammian and Pfaffian solutions for the (3+1)-dimensional generalized shallow water equation (the second member on the KP hierarchy). Recently, Yaning Tang [27] presented Pfaffian solutions and extended Pfaffian solutions to the (3+1)-dimensional Jimbo-Miwa equation (the second member on the KP hierarchy). In this paper, we have established Grammian and Wronskian solutions for the isospectral BKP equation (the second member on the BKP hierarchy). So the two equations not only has Pfaffian solutions but also has Grammian determinant solutions and Wronskian determinant solutions.

We would also like to repeat a problem related to this paper: which equation only has Grammian and Wronskian determinant solutions, which equation only has Pfaffian solutions, and which equation not only has Pfaffian solutions but also has Grammian and Wronskian determinant solutions. We expect to see more examples and a systematical theory finally. We hope that the current work is helpful for the future studies.

\section{Acknowledgements}

We express our sincere thanks to all the persons who have provided valuable suggestions to this paper. This work is supported by the National Natural Science Foundation of China (Grant Nos. 51109031, 51379033 and 
11201048), Doctoral Foundation of Ministry of Education of China (No. 20100041120037), the Fundamental Research Fund for the Central Universities, China (Grant Nos. DUT12LK34, DUT12LK52) and National Basic Research Program of China(2013CB036101,2010CB32700).

\section{References}

[1] M.J.Ablowitz, H.Segur, Solitons and the Inverse Scattering Transform. (SIAM, Philadelphia, 1981).

[2] R.K.Bullough, P.J.Caudrey, Solitons (Springer-Verlag, Berlin, 1980).

[3] D.J.Benny, J Math Phys 45 (1966) 52.

[4] M.Barriola, A.Vilenkin, Phys Rev Lett 63 (1989) 341.

[5] T.Colin Physica D 64 (1993) 215.

[6] D.Y.Chen, H.W.Xin and D.J.Zhang, Chaos, Solitons and Fractals 15 (2003) 761.

[7] E.Date, M.Jimbo, M.Kashiwara and T.Miwa, Phys.D 82 (1981) 343.

[8] E.Date, M.Jimbo, M.Kashiwara and T.Miwa, J.Phys.Soc.Jpn 50 (1981) 3183.

[9] N.C.Freeman, J.J.C.Nimmo, Soliton solutions of the Korteweg-de Vries and Kadomtsev-Petviashvili equations: The Wronskian technique, Physics.Letters.A. 95 (1983) 1-3.

[10] W.P.Hong, Phys Lett A 361 (2007) 520.

[11] R.Hirota, Phys.Rev.Lett. 27 (1971) 1192.

[12] R.Hirota, The direct method in soliton theory (Cambridge: Cambridge University Press, 2004).

[13] R.Hirota, Tang, Solutions of the Classical Boussinesq Equation and the Spherical Boussinesq Equation:The Wronskian Technique, J.Phys.Soc.Jpn. 55 (1986) 2137-2150.

[14] R.Hirota, J.Phys.Soc.Jpn 58 (1989) 2285.

[15] M.Jimbo, T.Miwa, Solitons and infinite dimensional Lie algebra, Res.I Math.Sci. 19 (1983) 943-1001.

[16] F.Kasch, Modules and Rings (Academic Press, London, New York, 1982).

[17] V.B.Matveev, M.A.Salle, Darboux Transformations and Solitons (Springer, Berlin, 1991).

[18] J.J.C.Nimmo and N.C.Freeman Physics.Letters. 96A (1983) 443-446.

[19] R.Radhakrishnan, M.Lakshmanan, Hietarinta J. Phys Rev E 56 (1997) 2213.

[20] C.Rogers, W.R.Shadwick, Bäcklund transformation and their application (Academic Press, New York, 1982).

[21] A.Ramani, Inverse scattering, ordinary differential equations of Painlevé type and Hirota's bilinear formalism, preprint, L.P.T.H.E., Universite Paris-Sud (1980).

[22] YePeeng Sun, HonWah Tam, Grammian solutions and pfaffianization of a non-isospectral and variablecoefficient Kadomtsev-Petviashvilli equation,J.Math.Anal.Appl. 343 (2008) 810-817.

[23] K.Sawada and T.Kotera, Prog.Theo.Phys 51 (1974) 1355.

[24] Wen-Rui Shan, Tian-Zhong Yan, Xing L, Min Li, Bo Tian, Analytic study on the Sawada-Kotera equation with a nonvanishing boundary condition in fluids, Commun Nonlinear Sci Numer Simulat 18 (2013) 1568-1575.

[25] M.Sato and M.Noumi, Soliton Equations and the Universal Grassmann Manifold, Sugaku Kokyuroku, Sophia Univ 18 (1984)[in Japanese].

[26] Tang Ya-Ning, Ma Wen-Xiu and Xu Wei, Grammian and Pfaffian solutions as well as Pfaffianization for a (3+1)-dimensional generalized shallow water equation, Chin.Phys.B 21 (2012) 070212.

[27] Yaning Tang, Applied Mathematical Modelling (Available online)(2013).

[28] Zhenya Yan, Multiple solution profiles to the higher-dimensional Kadomtsev-Petviashvilli equations via Wronskian determinant, Chaos, Solitons and Fractals. 33 (2007) 951-957. 